les parenchymateuses (probablement hépatiques) et non par les cellules hématopoïétiques (probablement dendritiques). Ces données permettent de reconstituer le scénario suivant: après inoculation dermique au cours d'une piqûre de moustique, les sporozoïtes migreraient dans le ganglion lymphatique de drainage, où ils seraient présentés par des cellules dendritiques à des lymphocytes $\mathrm{T} \mathrm{CD}^{+}$naïfs, permettant leur activation. Ces cellules activées migreraient alors dans la circulation et le foie où elles seraient alors capables de reconnaître des antigènes parasitaires présentés directement par l'hépatocyte, et non plus par une cellule présentatrice d'antigène professionnelle. Bien que la réponse immunologique induite ne soit pas complète, elle pourrait permettre de contenir l'infection parasitaire [2]. Ces données nouvelles vont certainement avoir des conséquences déterminantes sur la façon de concevoir la vaccination antipalustre. Elles constituent un argument supplémentaire pour une approche

\title{
NOUVELLE
}

\section{L'autophagie remplaçant de luxe du protéasome}

Julien Puyal, Vanessa Ginet, Anne Vaslin, Peter G.H. Clarke

> La machinerie protéolytique de la cellule est composée de deux mécanismes majeurs: la dégradation protéasomique et l'autophagie. Le premier est spécifique aux protéines à courte durée de vie qui, lorsqu'elles sont anormales ou présentes en quantité excessive, subissent une modification post-traductionnelle, la poly-ubiquitinylation, et sont ensuite reconnues et dégradées par le protéasome $[1,2]$. Le deuxième est la macroautophagie (appelée ci-après autophagie), mécanisme considéré comme moins sélectif, qui assure la régulation et l'élimination de protéines à longue durée de vie et celle d'organites cellulaires altérés (comme les mitochondries) [3, 4]. Dans cette voie, de grosses vacuoles, appelées autophagosomes, séquestrent une portion de cytosol contenant les protéines et organites à éliminer, puis fusionnent avec les lysosomes contenant des protéases qui vont assurer la dégradation de leur contenu.

Dans certaines conditions (mutations, stress oxydatif, âge...) une altération du repliement des protéines accompagnée d'un défaut de leur élimination peut entraîner leur agrégation irréversible et la formation d'inclusions insolubles précédant la mort cellulaire. Ce phénomène est une caractéristique commune à de nombreuses maladies neurodégéneratives (agrégats de $\beta$-amyloïde dans la maladie d'Alzheimer, d' $\alpha$-synucléine pour la maladie de Parkinson et d'Huntingtine pour la maladie de Huntington) [5]. Le contrôle de la conformation des protéines est donc indispensable au maintien de l'homéostasie cellulaire et I'on peut considérer qu'un défaut dans la dégradation des protéines non fonctionnelles, qui survient notamment avec l'âge, peut être impliqué dans le développement de certaines maladies neurodégénératives.

\section{L'autophagie constitutive : rôle} complémentaire à celui du protéasome en conditions physiologiques

Le rôle de l'autophagie constitutive dans les neurones n'a été que récemment mis en évidence grâce à des vaccinale fondée sur l'inoculation intradermique de parasites entiers ou tout du moins d'antigènes présentés à la fois sur le sporozoïte et sur le foie infecté. $\diamond$ Lymph nodes: first barrier against Plasmodium

\section{RÉFÉRENCES}

1. Chakravarty S, Cockburn IA, Kuk S, et al. $C D 8^{+} T$ lymphocytes protective against malaria liver stages are primed in skin-draining lymph nodes. Nat Med 2007 ; 13 : 1035-41.

2. Good MF, Doolan DL. Malaria's journey through the lymph node. Nat Med 2007 ; 13 : 1023-4.
Département de Biologie Cellulaire et de Morphologie (DBCM), Université de Lausanne, Rue du Bugnon 9, 1005 Lausanne, Suisse.

Peter.Clarke@unil.ch

travaux sur des souris génétiquement modifiées n'exprimant pas certains gènes autophagiques essentiels (ATG 5 et 7) $[6,7]$. Ces souris présentent une accumulation progressive de protéines ubiquitinylées diffuses (solubles) dans les neurones associée à des symptômes sévères de neurodégénérescence. Dans les conditions basales, l'autophagie permettrait donc d'éliminer les protéines ubiquitinylées solubles non dégradées par la voie du protéasome et aurait ainsi une fonction complémentaire à celle du protéasome dans la prévention de la neurodégénerescence. L'ubiquitine ne semble donc pas spécifique au protéasome et pourrait être un signal de dégradation commun au protéasome et à l'autophagosome, permettant ainsi une communication entre ces deux voies. Si tel est le cas, ce lien pourrait être fait notamment grâce au complexe multiprotéique p62/ sequestosome-1 (SQSTMI), qui possède un domaine d'interaction avec la protéine autophagosomique LC3 (ATG8) et un autre avec l'ubiquitine [8]. 
L'autophagie induite:

rôle compensatoire face

aux défaillances du protéasome

en conditions pathologiques

Dans les maladies neurodégénératives, la formation des agrégats protéiques est très souvent associée à une augmentation de l'autophagie et à une diminution de l'activité du protéasome [9]. Ces agrégats auraient d'abord une fonction cytoprotectrice en séquestrant les protéines diffuses (toxiques) et ils stimuleraient également l'autophagie. L'autophagie ainsi induite jouerait un rôle important dans les premiers stades de la neurodégénérescence afin de lutter contre l'accumulation des protéines non fonctionelles et la formation d'inclusions. De plus, la stimulation pharmacologique de l'autophagie dans des lignées de cellules ou des animaux porteurs de mutations induisant des maladies neurodégénératives est suffisante pour éliminer les agrégats protéiques. Ces études suggèrent donc une activité compensatrice de l'autophagie par rapport à celle du protéasome, ce qui a été confirmé par un récent travail effectué chez la drosophile. En effet, l'inhibition de manière génétique de l'activité du protéasome au niveau des yeux de la drosophile est toxique mais la stimulation en parallèle de l'autophagie (par un agent pharmacologique) permet de prévenir cette toxicité [10]. La stimulation de l'autophagie est donc suffisante pour prévenir la mort cellulaire associée à l'absence d'activité du protéasome et pourrait ainsi prendre en charge la dégradation de l'ensemble des protéines en se substituant au protéasome. Cependant, il reste à démontrer que cette

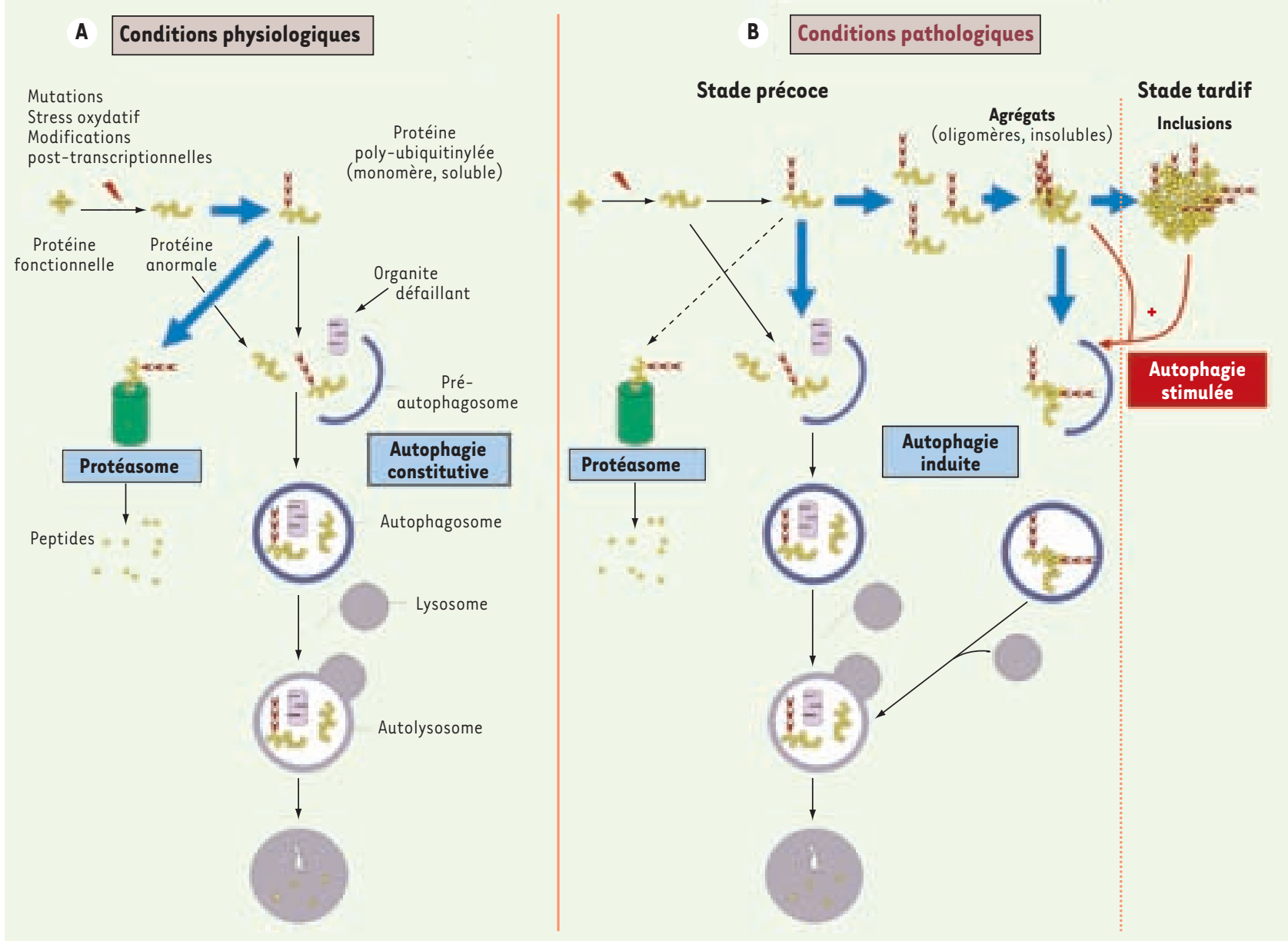

Figure 1. Autophagie et maladies neurodégénératives. A. Conditions physiologiques. Une protéine neuronale anormale (conformation inadéquate) va être polyubiquitinylée afin d'être dégradée par le protéasome en peptides, puis en acides aminés par les peptidases nucléaires et cytosoliques. L'autophagie, quant à elle, prend en charge la dégradation des organites défaillants ainsi que celle de certaines protéines (à longue durée de vie ou non dégradées par le protéasome). B. Conditions pathologiques. En cas de maladies neurodégénératives, des agrégats de protéines anormales vont se former (suite à une diminution de l'activité du protéasome par exemple). La présence de ces agrégats entraîne une augmentation de l'autophagie (autophagie induite) qui, dans les premiers stades de la maladie, est assez efficace pour assurer leur élimination. À un stade plus avancé, l'autophagie induite n'est plus suffisante et des inclusions protéiques se forment. À ce stade, la stimulation de l'autophagie (par un agent pharmacologique) pourrait s'avérer efficace pour éliminer les inclusions et ainsi lutter contre la neurodégénérescence. 
action protectrice de l'autophagie en cas de toxicité secondaire à l'inhibition du protéasome est due à une élimination accrue des protéines et non pas à une augmentation de la clairance des mitochondries (éliminant ainsi la libération de molécules pro-apoptotiques).

\section{Perspectives : stimuler l'autophagie pour éviter la neurodégénerescence} Chez l'homme l'autophagie induite dans les maladies neurodégénératives n'est malheureusement pas suffisante pour assurer l'élimination totale des agrégats. Alors pourquoi ne pas administrer un agent stimulateur de l'autophagie dès l'apparition des premiers symptômes chez les patients atteints de la maladie de Parkinson ou d'Alzheimer? Une telle stratégie neuroprotectrice peut parâ̂tre séduisante mais il faut prendre en considération qu'une stimulation exces- sive de l'autophagie peut aussi entraîner la mort de la cellule, nommée mort cellulaire de type II ou autophagique. $\varepsilon n$ effet, une activation excessive de l'autophagie visant à éliminer les agrégats de protéines anormales pourrait aboutir à une dégradation incontrôlée affectant des composants cellulaires essentiels à la survie cellulaire. $\diamond$

Autophagy

as a proteasomal substitute

\section{REMERCIEMENTS}

Le travail de notre laboratoire sur l'autophagie est soutenu par le subside 3100A0-113925 du Fonds national suisse.

\section{RéFÉRENCES}

1. Andermarcher $\varepsilon$, Bossis G, Farras R, et al. La dégradation protéasomique : de l'adressage des proteins aux nouvelles perspectives thérapeutiques. Med Sci (Paris) 2005 ; $21: 141-9$.
2. Pickart CM. Back to the future with ubiquitin. Cell 2004 ; 116 : 181-90.

3. Codogno $P$. Les gènes ATG et la macro-autophagie. Med Sci (Paris) 2004 ; 20 : 734-6.

4. Klionsky DJ, Emr SD. Autophagy as a regulated pathway of cellular degradation. Science 2000 ; $290: 1717-21$.

5. Ross CA, Poirier MA. Protein aggregation and neurodegenerative disease. Nat Med 2004; 10 (suppl) : S10-7.

6. Hara T, Nakamura K, Matsui M, et al. Suppression of basal autophagy in neural cells causes neurodegenerative disease in mice. Nature 2006 ; $441: 885-9$.

7. Komatsu M, Waguri S, Chiba T, et al. Loss of autophagy in the central nervous system causes neurodegeneration in mice. Nature $2006 ; 441: 880-4$

8. Pankiv S, Clausen TH, Lamark T, et al. p62/SDSTMl binds directly to Atg8/LC3 to facilitate degradation of ubiquitinated protein aggregates by autophagy. J Biol Chem 2007 ; 282 : 24131-45.

9. Rubinsztein DC. The roles of intracellular proteindegradation pathways in neurodegeneration. Nature 2006 ; 443 : 780-4.

10. Pandey UB, Nie Z, Batlevi Y, et al. HDAC6 rescues neurodegeneration and provides an essential link between autophagy and the UPS. Nature 2007 ; $447: 859-63$.

\section{NOUVELLE}

\section{Le diabète est tombé sur un os...}

Cyrille B. Confavreux, Mathieu Ferron
HHSC 1614, Department of Genetics and Development, Columbia University, New York, Ny 10032 États-Unis. cc2794@columbia.edu
> Outre ses fonctions mécaniques et son rôle dans l'homéostasie du calcium, le tissu osseux sécrète une hormone, l'ostéocalcine, impliquée dans la régulation du métabolisme énergétique [1].

Que l'obésité protège de l'ostéoporose et que l'anorexie la favorise suggère depuis longtemps l'existence d'un lien entre les métabolismes osseux et énergétique. De même, les souris ob/ob qui sont hyperphagiques et obèses par absence de leptine, hormone sécrétée par les adipocytes, ont une masse osseuse élevée [2, 3]. Une première série d'expériences a démontré que la leptine stimulait le système nerveux sympathique via les neurones hypothalamiques et freinait l'activité des ostéoblastes, cellules responsables de la formation osseuse et exprimant des récepteurs $\beta 2$ adrénergiques [4]. Cette découverte de la régulation centrale de la masse osseuse a

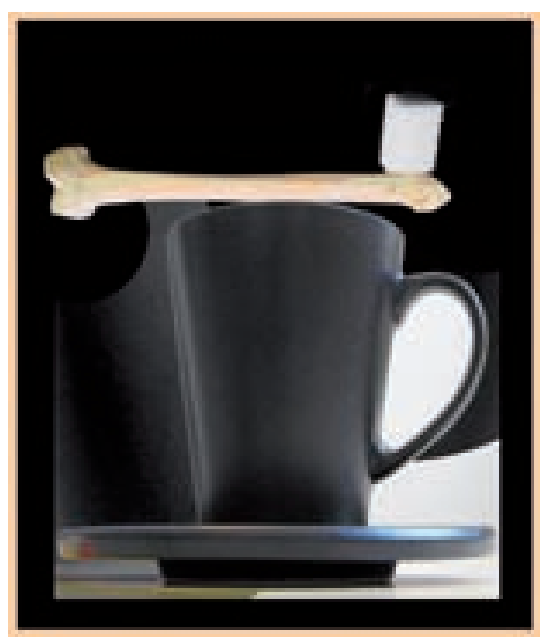

établi l'existence d'un lien hormonal entre tissu adipeux et tissu osseux [5]. Comme la physiologie endocrinienne repose sur l'existence de boucles de rétrocontrôle, ces résultats suggéraient que les ostéoblastes pouvaient, à leur tour, sécréter des molécules régulant le métabolisme énergétique. La recherche de telles hormones a reposé sur une approche génétique. Très peu de gènes sont spécifiquement exprimés dans les ostéoblastes. L'un d'eux, Esp (Ptprv), code pour une phosphatase exprimée uniquement dans les ostéoblastes et les cellules de Sertoli. Sa fonction a été étudiée chez la souris après l'inactivation du gène soit dans toutes les cellules soit ciblée seulement dans les ostéoblastes [6]. 